\title{
Quantum fluids in nanoporous media-Effects of the confinement and fractal geometry
}

\author{
TAYURSKII Dmitrii* \& LYSOGORSKIY Yury \\ Department of Physics, Kazan Federal University, 18 Kremlevskaya st., Kazan 420008, Russia
}

Received May 17, 2011; accepted June 27, 2011

\begin{abstract}
The complex behavior of such quantum fluids like liquid ${ }^{4} \mathrm{He}$ and liquid ${ }^{3} \mathrm{He}$ in nanoporous media is determined by spatial quantization because of geometrical confinement as well as by significant contribution from the surface atoms. In the present report we will review the procedure, results and discuss the issues for fractionalized nonextensive hydrodynamical approach to describe the properties of quantum fluids inside nanopores and propose consideration of strong correlated quantum liquid by means of fractionalized Schrödinger equation.
\end{abstract}

superfluid, aerogel, nanoscale physics, fractional derivative

Citation: Tayurskii D, Lysogorskiy Y. Quantum fluids in nanoporous media — Effects of the confinement and fractal geometry. Chinese Sci Bull, 2011, 56: 3617-3622, doi: $10.1007 / \mathrm{s} 11434-011-4761-\mathrm{z}$

The zero-point energy of atoms of two stable isotopes of helium $-{ }^{4} \mathrm{He}$ and ${ }^{3} \mathrm{He}$ - is high enough to prevent their solidification even at extremely low temperatures without application of external pressure. So they belong to the class of quantum fluids with strong correlations between atoms but their behavior is quite different at low temperatures. The first one represents a Bose-system and shows superfluid transition at $2.17 \mathrm{~K}$ while atoms of ${ }^{3} \mathrm{He}$ are governed by Fermi-statistics and superfluid transition can be observed only at much lower temperatures (about $1 \mathrm{mK}$ ) when the pairing of two atoms occurs. But even at liquid helium temperatures (1.5-4.2 K) the effects of quantum statistics for ${ }^{3} \mathrm{He}$ atoms become pronounceable especially in nanoscale confinement (nanoporous media, thin adsorbed layers on solid substrates) [1-3] and in the presence of nanoscale disorder induced for example by silica aerogel strands [4]. In recent years the problem of correct description of quantum fluids in the confined geometry at nanoscale length has emerged [5-8]. It has been recognized that the quantum fluids at these circumstances can be considered as a new state of quantum matter due to close values between characteristic lengths for these quantum liquids and the size of geometrical confinement and significant contri-

*Corresponding author (email: dtayursk@gmail.com) bution from the surface atoms. So one has to apply new physics to describe such systems with taking into account their complex nature. For example, last two years the attempts to develop the fractionalized two-fluid hydrodynamics for nanoporous media with fractal dimensions have been made $[9,10]$. The actuality of such new hydrodynamics becomes very clear because of numerous studies of quantum liquids inside nanoporous media [11,12] as well as because of last developments in chemical synthesis of aerogels with different network of strands (from fibrous to globular one [13]). One of the interesting obtained results is that density waves (the first sound) and temperature waves (the second sound) become strongly coupled even in the absence of viscosity, so it is purely geometric effect of fractal space of nanopores $[9,10]$.

In this paper we will review the procedure, results and discuss the issues for this approach.

\section{Theoretical models for bulk superfluid helium-4}

There are several approaches to describe the behavior of bulk superfluid helium-4. For example, two-fluid model [14, 15], 
the microscopic description based on the Gross-Pitaevskii equation $[16,17]$ and others.

\subsection{Two-fluid model}

The most known model is a two-fluid model (TFM) in the framework of which superfluid helium with density $\rho$ is considered as a two component system: an uncondensed, normal component with density $\rho_{n}$ with velocity $\boldsymbol{v}_{n}$ and a condensed, superfluid component characterized by density $\rho_{s}=$ $\rho-\rho_{n}$ with velocity $\boldsymbol{v}_{s}$. Without dissipative terms one finds the following system of hydrodynamical equations (so-called Landau-Khalatnikov equations $[14,15]$ ):

$$
\begin{aligned}
\frac{\mathrm{d} \rho}{\mathrm{d} t}+\operatorname{div}\left(\rho_{n} \boldsymbol{v}_{n}+\rho_{s} \boldsymbol{v}_{s}\right) & =0, \\
\frac{\partial}{\partial t}\left(\rho_{n} \boldsymbol{v}_{n}+\rho_{s} \boldsymbol{v}_{s}\right)_{i}+\frac{\partial}{\partial x_{k}} \Pi_{i k} & =0, \\
\frac{\mathrm{d} S}{\mathrm{~d} t}+\operatorname{div} S \boldsymbol{v}_{n} & =0, \\
m \frac{\partial \boldsymbol{v}_{s}}{\partial t}+m\left(\boldsymbol{v}_{s} \nabla\right) \boldsymbol{v}_{s}+\nabla \mu & =0 .
\end{aligned}
$$

The entropy is denoted by $S$, the mass of an atom by $m$, the pressure by $p$ and the chemical potential by $\mu$. In eq. (2) the sum over the index $k$ is assumed and the stress tensor $\Pi_{i k}$ is given by

$$
\Pi_{i k}=\rho_{n} v_{n i} v_{n k}+\rho_{s} v_{s i} v_{s k}+p \delta_{i k}
$$

One can obtain from these equations that there are two type of collective motion: motion of the fluid where both components move in phase is called first (ordinary) sound, while second sound is associated with out of phase motion of the two components (Figure 1). The above TFM equations describe the flow properties of superfluid ${ }^{4} \mathrm{He}$ in the bulk of the liquid. They are not valid in the immediate proximity of walls and free surfaces, where the effects of so-called "healing" are encountered. Moreover, Landau-Khalatnikov equations cannot be used in the vicinity of $\lambda$ point, where large variations of superfluid density in space as well as in time may occur. To resolve this problem Ginzburg and Pitaevskii have proposed to use their phenomenological theory with a complex order parameter [18]. Later this approach has been extended and modified by Khalatnikov [19]. The hydrodynamic theory of He-II incorporating the effects of quantum healing and relaxation has been developed by Hills and Roberts [17,20]. Later Guerst [21] has proposed the general phenomenological theory of superfluid ${ }^{4} \mathrm{He}$ unifying and extending LandauKhalatnikov, Hills-Roberts and Ginzburg-Pitaevskii theories.

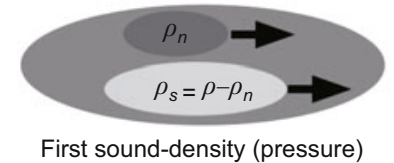

oscillations

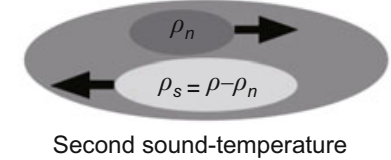

(entropy) oscillations
Figure 1 Schematic view of sound modes in superfluid helium-4 in the framework of two-fluid hydrodynamic model.

\subsection{Nonlinear Schrödinger equation}

If the short range pair interaction between particles can be written as $U\left(x-x^{\prime}\right)=U_{0} \delta\left(x-x^{\prime}\right)$, where $N_{0}=\int|\Psi|^{2} \mathrm{~d}^{3} x$ denotes the number of particles in condensate and $V_{0}$ stands for total condensate volume, then one can obtain nonlinear Schrödinger equation [16] in the following form:

$$
i \frac{\partial \Psi}{\partial t}=-\frac{1}{2} \nabla^{2} \Psi+|\Psi|^{2}-\Psi .
$$

Here average density $\rho_{0}=N_{0} / V_{0}$ is supposed to be equal to unit, the units of length and time are defined as $\hbar / \sqrt{\rho_{0} U_{0}}$ and $\hbar /\left(\rho_{0} U_{0}\right)$, respectively. Strictly speaking this equation is valid only for absolute zero temperature, when all atoms of $\mathrm{He}-\mathrm{II}$ are in the condensate state and depletion of this state is negligible so one can write for the condensate wave function $\Psi(x, t)=\sqrt{\rho(x, t)} \mathrm{e}^{i \theta(x, t)}$. Substituting last equation for $\Psi(x, t)$ and separating real and imaginary parts in eq. (6) one can obtain

$$
\begin{aligned}
& \frac{\partial \rho}{\partial t}+\nabla \cdot \rho v=0 \\
& \frac{\partial v}{\partial t}+\nabla \frac{v^{2}}{2}+\frac{1}{\rho} \nabla \frac{\rho^{2}}{2}=-\nabla\left(\frac{(\nabla \rho)^{2}}{8 \rho^{2}}-\frac{\nabla^{2} \rho}{4 \rho}\right),
\end{aligned}
$$

where $v \equiv \nabla \theta$. Except for high-order derivatives in right hand side of eq. (8), which can be omitted in hydrodynamical limit, the system of eqs. (7) and (8) is equivalent to Euler equations for non-rotating ideal fluid with a pressure defined as $p(\rho) \equiv \rho^{2} / 2$. The pressure depends only on density $\rho$ because the fluid is considered at absolute zero temperature.

To consider dynamics of He-II at nonzero temperature, Hills and Roberts have improved TFM [17] by introducing additional terms which were proportional to the gradient of superfluid component. In this case eq. (4) can be rewritten as

$$
\frac{\partial \boldsymbol{v}_{s}}{\partial t}+\nabla\left(\frac{\boldsymbol{v}_{s}^{2}}{2}+\mu\right)=\nabla\left(\eta\left(\rho_{s}\right) \nabla^{2} \rho_{s}+\frac{1}{2} \frac{\mathrm{d} \eta}{\mathrm{d} \rho_{s}}\left(\nabla \rho_{s}\right)^{2}\right),
$$

where $\eta\left(\rho_{s}\right)$ denotes some function to be defined; stress tensor has to be modified and rewritten as

$$
\begin{aligned}
\Pi_{i j} \equiv & \rho_{n} v_{n i} v_{n j}+\rho_{s} v_{s i} v_{s j}+\eta \nabla_{i} \rho_{s} \nabla_{j} \rho_{s} \\
& +\left(p-\eta \rho_{s} \nabla^{2} \rho_{s}-\frac{1}{2} \frac{\rho_{s} \eta}{\rho_{s}}\left(\nabla \rho_{s}\right)^{2}\right) \delta_{i j} .
\end{aligned}
$$

New terms including spatial derivatives of $\rho_{s}$ are responsible for macroscopic quantum effects, like healing length [17].

\subsection{Correlated density matrix theory}

Another way to describe a such quantum strongly correlated system like superfluid helium is correlated density matrix [22] theory that provides the method of choice to analyze the microscopic structure of strongly correlated quantum fluids in thermal equilibrium. This theory allows to study the combined effects of dynamic correlations and quantum-statistical 
effects in correlated Bose and Fermi liquids. The final outcome of the correlated density matrix theory is that the properties of strongly correlated quantum fluids at finite temperatures can be studied with reference to the background gas of renormalized free particles (bosons or fermions). The mass of such a renormalized boson or fermion depends in a specific form on temperature, bulk particle number density of the many-body system, and eventually on momentum.

\subsection{Extended irreversible thermodynamics}

Recently it was shown $[23,24]$ that the main properties of superfluid helium, both in the absence and in the presence of dissipation, can be explained using a mono-fluid model based on the extended irreversible thermodynamics where four fields, namely density, temperature, velocity, and heat flux are involved as independent fields. This model is able to explain the propagation of the two sounds that are typical of helium II, and the attenuation calculated for such sounds is in agreement with the experiment results. The model is more general than the standard TFM because it allows that a small amount of entropy is associated with helium when it flows through a porous medium (see section 2 ).

\section{Aerogel}

Silica aerogels are synthesized via a sol-gel process and hypercritical drying which enable production of tenuous solids with porosity $\phi$ as large as $99.8 \%$ and unique acoustic properties. Silica aerogel are known to be good examples of fractal materials. A revealed by small-angle X-ray-scattering (SAXS) experiments or small-angle neutron-scattering (SANS) experiments, they are made of a disorder, but homogeneous, array of connected fractal clusters resulting from the aggregation of primary particles. The analysis of the wavevector dependence of the scattering intensity $I(q)$ has permitted the determination of two characteristic length scales which are the average size $a \approx 10 \AA$ of the particles and the average size of the clusters $\xi \approx 100 \AA$. At length scales from $a$ to $\xi$ silica aerogels show a fractal behavior [25].

The computational confirmation for cluster structure of aerogel has been obtained by modeling as well as by the geometrical analysis of the diffusion limited cluster-cluster aggregation $[26,27]$.

Also it has been demonstrated [7] that it is long-correlated structure of aerogel that makes an essential influence on liquid ${ }^{4} \mathrm{He}$ behavior near $\lambda$-point.

\subsection{Behavior of ${ }^{4} \mathrm{He}$ inside aerogel}

There has been considerable interest in the behavior of superfluid ${ }^{4} \mathrm{He}$ in the presence of a random disorder induced by highly open porous media, like aerogel. Understanding the results of acoustic experiments is important when dealing with porous media. Use of liquid ${ }^{4} \mathrm{He}$ offers unique advantages due to the existence of the superfluid phase with more than one sound mode. In a porous media where the normal component is clamped by its viscosity and only the superfluid component can move, fourth sound (relative motion of the superfluid and normal fluids) propagates and can be used to determine the superfluid fraction.

The high-porosity aerogels are so soft that the aerogel matrix clamped with normal fluid is caused to move as by the pressure and temperature gradients, unlike other porous media. This results in sound mode intermediate between first and fourth sound [28] and a second-sound-like mode [29] (Figure 2). In this case, proposed by Biot the theory of acoustic propagation in porous, fluid filled, macroscopically homogeneous and isotropic media [30], is not applicable.

It is very interesting to study the possible influence of geometrical confinement with fractal dimensionality on the flow properties of superfluid ${ }^{4} \mathrm{He}$ in the framework of TFM. In order to shed a light on geometrical factor itself one can neglect here by quantum healing and any dissipative processes.

\subsection{Nonextensivity}

So hereafter we suppose that effectively aerogel can be considered as a cluster with a fractal mass dimension [31] the nanopores of which are filled in by liquid helium.

Note that thermodynamic limit conditions are violated for helium atoms inside nanopores because of a huge inner pore surface of aerogel (up to $3000 \mathrm{~m}^{2} / \mathrm{g}$ for an aerogel with density $2 \mathrm{mg} / \mathrm{cm}^{3}$ ). Namely the ratio of total number of helium atoms $N$ to the total cluster volume $V$ is not constant at $N, V \rightarrow \infty$ and nonextensivity of physical properties takes place for helium atoms in two nanopores. In this case the methods of non-extensive thermodynamics [32] should be applied to construct the two-fluid hydrodynamic model and non-extensive entropy like Tsallis entropy should be introduced. We have to note here that beyond thermodynamic limit even the usual Boltzmann-Gibbs entropy becomes nonadditive, but the additivity is restored when thermodynamic limit conditions are taken [33].

\section{Fractionalized and nonextensive hydrody- namical model}

In general case a macroscopic quantity $Q(A, B)$ associated

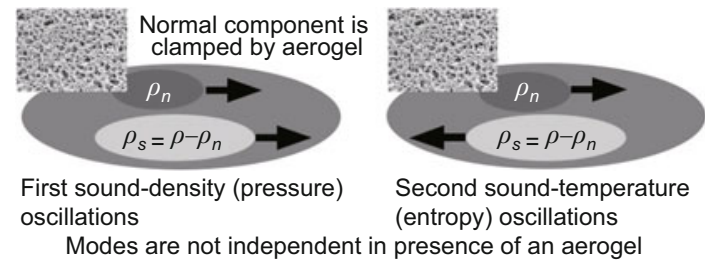

Figure 2 Schematic view of sound modes in superfluid helium-4 inside an aerogel. The aerogel matrix is clamped with normal fluid so the first and the second sound are not independent now and new sound modes appear - a sound mode intermediate between first and fourth sound and a secondsound-like mode. 
with the total system may be expressed in terms of the same quantity associated with the subsystems, $Q(A)$ and $Q(B)$ [34]:

$$
Q(A, B)=f_{\lambda_{Q}}[Q(A), Q(B)],
$$

where $f_{\lambda_{Q}}$ is a symmetric bivariate function depending on a constant $\lambda_{Q}$. Of course, for given quantity $Q$ there exist many functions which satisfy the composability property (11). However, additional assumptions drastically reduce their number. For example, the thermodynamic equilibrium may be used as a constraint on the form of $f_{\lambda_{Q}}$ in eq. (11). For energy $E_{\lambda}$ [35] and entropy $S_{q}$ [36] of helium inside nanopores it leads to

$$
\begin{aligned}
& E_{\lambda}(A+B)=E_{\lambda}(A)+E_{\lambda}(B)+\lambda E_{\lambda}(A) E_{\lambda}(B), \\
& S_{q}(A+B)=S_{q}(A)+S_{q}(B)+q S_{q}(A) S_{q}(B),
\end{aligned}
$$

where $\lambda$ and $q$ are parameters of nonextensivity and are determined by the properties of system. Suppose that a local equilibrium between liquid ${ }^{4} \mathrm{He}$ in different nanopores takes place. So we can re-define such thermodynamic quantities as temperature and pressure in the following manner [37]:

$$
\begin{aligned}
& T_{\text {phys }}=\frac{1+q S_{q}}{1+\lambda E_{\lambda}}\left(\frac{\partial E_{\lambda}}{\partial S_{q}}\right)_{V}, \\
& p_{\text {phys }}=\frac{T_{\text {phys }}}{1+q S_{q}}\left(\frac{\partial S_{q}}{\partial V}\right)_{E} .
\end{aligned}
$$

Further, it is possible to introduce the spatial pressure and density distributions in fractal cluster as it has been made in our previous work [9]:

$$
\begin{aligned}
& p_{\text {phys }}(r)=p_{f}(r) \chi_{p}(r), \\
& \rho_{\text {phys }}(r)=\rho_{f}(r) \chi_{\rho}(r),
\end{aligned}
$$

where $r$ is distance from center of fractal cluster, $p_{f}(r)$ is distribution of pressure in pore, $\chi_{p}(r)$ is a fractal factor-function. In the case of Euclidean space with $D=3$ this factor-function should be equal to unit: $\chi_{p}(r) \equiv 1$. Such kind of fractionalization procedure can be applied to any thermodynamic quantity $A$, i.e.

$$
\begin{gathered}
A=A_{f}(X, Y, Z, \ldots) \chi_{A}(r), \\
\lim _{D \rightarrow 3} \chi_{A}=1 .
\end{gathered}
$$

From the defined thermodynamic quantities $(14,15)$ and eq. (16) the fractal factor-functions for energy, entropy and temperature are derived:

$$
\begin{aligned}
& \chi_{E}=\chi_{S}=\frac{\chi_{p}}{1+\lambda E_{f}\left(1-\chi_{p}\right)}, \\
& \chi_{T}=\frac{1+q S_{f} \chi_{S}}{1+q S_{f}} \frac{1+\lambda E_{f}}{1+\lambda E_{f} \chi_{E}} .
\end{aligned}
$$

Because all thermodynamic quantities should be expressed in terms of physical (observable) variables, one can propose the following definition of the generalized free energy:

$$
F=E-T_{\text {phys }} S_{\text {phys }}=E-\left(\frac{\partial E}{\partial S_{\text {phys }}}\right) S_{\text {phys }},
$$

which represents no more than the Legendre transformation. So the entropy differential equals to

$$
\mathrm{d} S_{\text {phys }}=\frac{1+\lambda E_{\lambda}}{1+q S_{q}} \mathrm{~d} S_{q}
$$

In the first order with respect to $\lambda E_{f}$ and $q S_{q}$ the entropy can be written as

$$
\begin{aligned}
S_{\text {phys }} & =\frac{1}{q} \ln \left(1+q S_{q}\right)+\lambda \int \frac{E_{\lambda}\left(S_{q}\right) \mathrm{d} S_{q}}{1+q S_{q}} \\
& \approx S_{q}-q S_{q} \frac{S_{q}}{2}+\lambda \int E_{\lambda} \mathrm{d} S_{q} .
\end{aligned}
$$

Finally the fractal factor-function for $S_{\text {phys }}$ is

$$
\chi_{S p}=\chi_{S}+\chi_{p}\left(\chi_{p}-1\right)\left(\frac{\lambda E_{f}-q S_{f}}{2}+\lambda H\right),
$$

where $H=1 / S_{f} \int E_{f} \mathrm{~d} S_{f}$. After substitution the fractionalized thermodynamical quantities into TFM one can derive the main system of equations for the fractionalized two-fluid hydrodynamic model:

$$
\begin{array}{r}
\frac{\partial \rho_{f}}{\partial t} \chi_{p}+\operatorname{div}\left(\rho_{s f} \chi_{p} v_{s}+\rho_{n f} \chi_{p} v_{n}\right)=0, \\
\frac{\partial \rho_{f} \sigma_{f}}{\partial t} \chi_{s p}+\operatorname{div}\left(\rho_{f} \sigma_{f} \chi_{s p} v_{n}\right)=0, \\
\rho_{s f} \chi_{p} \frac{\partial v_{s}}{\partial t}=-\frac{\rho_{s f}}{\rho_{f}} \nabla\left(p_{f} \chi_{p}\right)+\rho_{s f} \sigma_{f} \chi_{p} \chi_{\sigma} \nabla\left(T_{f} \chi_{T}\right), \\
\rho_{n f} \chi_{p} \frac{\partial v_{n}}{\partial t}=-\frac{\rho_{n f}}{\rho_{f}} \nabla\left(p_{f} \chi_{p}\right)-\rho_{s f} \sigma_{f} \chi_{p} \chi_{\sigma} \nabla\left(T_{f} \chi_{T}\right),
\end{array}
$$

where $\chi_{\sigma}=1+\left(\chi_{p}-1\right)\left(\frac{3 \lambda E_{f}-q S_{f}}{2}+\lambda H\right)$. From eqs. (26)-(29) two equations for waves of pressure and temperature follow

$$
\begin{aligned}
\frac{1}{u_{1}^{2}} \frac{\partial^{2} p_{f}}{\partial t^{2}}= & \nabla^{2} p_{f}+2 \frac{\nabla \chi_{p}}{\chi_{p}} \nabla p_{f}+\frac{\nabla^{2} \chi_{p}}{\chi_{p}} p_{f} \\
\frac{1}{u_{2}^{2}} \frac{\partial^{2} T_{f}}{\partial t^{2}}= & \nabla^{2} T_{f}\left(1+\left(\chi_{p}-1\right) M\right) \\
& +\nabla T_{f}\left(M \nabla \chi_{p}-(1-M) \frac{\nabla \chi_{p}}{\chi_{p}}\right) \\
& +T_{f}\left(q S_{f}-\lambda E_{f}\right)\left(\nabla^{2} \chi-\frac{\left(\nabla \chi_{p}\right)^{2}}{\chi_{p}}\right) \\
& +\frac{1}{u_{2}^{2}} \frac{N \sigma_{f}}{\rho_{f}}\left(\frac{\partial T_{f}}{\partial \sigma_{f}}\right)_{\rho_{f}}\left(\nabla \chi_{p} \nabla p_{f}+\frac{\left(\nabla \chi_{p}\right)^{2}}{\chi_{p}} p_{f}\right)
\end{aligned}
$$

where $u_{1}^{2}=\left(\partial p_{f} / \partial \rho_{f}\right)_{S_{f}}, u_{2}^{2}=\rho_{s f} \sigma_{f}^{2} / \rho_{n f}\left(\partial T_{f} / \partial \sigma_{f}\right)_{p_{f}}$ are the squared first and second sound velocities respectively and $M=\left(\lambda E_{f}+q S_{f}\right) / 2+\lambda H, N=\left(3 \lambda E_{f}-q S_{f}\right) / 2+\lambda H$. In Figure 3 the profiles for pressure wave and for the temperature wave induced by it are shown. It is seen from eq. (31) that the coupling between pressure and temperature waves appears even in the absence of ${ }^{4} \mathrm{He}$ viscosity and aerogel sceleton inertia, 


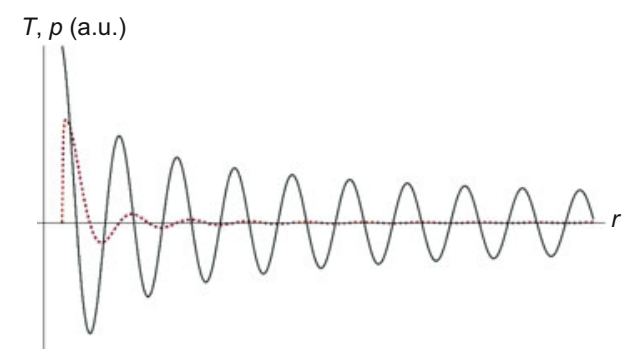

Figure 3 Profile for pressure wave (solid line) and the temperature wave induced by it (dashed line).

which is undoubtedly the effect of not only fractional dimensionality of nanopore space, but also of the nonextensive nature of thermodynamical quantities for He-II inside nanopores.

\section{Fractional microscopical approach}

In contrast to purely phenomenological two-fluid model, as mentioned in Section 1.2, one can start the study of problem from microscopical point of view. In this section the fractional Schrödinger equation will be considered and its application to superfluid at nanoscale will be motivated.

\subsection{Fractional Schrödinger equation}

An alternative point of view on quantum mechanical motion is the so-called Feynman formalism of quantum mechanics, which is focused on the concept of trajectory, and the particle can move along any possible trajectories. To move a particle from point $\mathrm{A}$ to point $\mathrm{B}$ one must take into account the contribution of all possible trajectories with the corresponding weight (complex factor). Possible trajectories resemble Brownian trajectory of a free particle and have a fractal dimension of $\alpha=2$.

De Broglie thermal wavelength for the helium atom inside the aerogel at temperatures of about $1 \mathrm{~K}$ is about $10 \AA$, which is in proper relation with the characteristic length scale of the fractal structures formed aerogel. Thus, some quantummechanical trajectory of the helium atom will be forbidden due to the influence of the structure of aerogel. Realized trajectory will resemble the motion of a Brownian particle in a porous medium, where the mean square displacement depends on time as $<x^{2}>\propto t^{2 / \alpha}$. This phenomenon is called subdiffusion. To describe this type of diffusion an equation with fractional Riesz derivative is used. Probability density function for such case is written in terms of Levy function, which is a generalization of the Gaussian distribution.

Laskin proposed to generalize the Feynman's path integrals to an arbitrary fractal dimension of trajectories $\alpha$ [38]. From this type of path integrals one can obtain fractional Schrödinger equation which is written as

$$
i \hbar \frac{\partial \psi(\boldsymbol{r}, t)}{\partial t}=D_{\alpha}(\hbar \nabla)^{\alpha} \psi(\boldsymbol{r}, t)+V(\boldsymbol{r}, t) \psi(\boldsymbol{r}, t) .
$$

Here Riesz fractional derivative is introduced as

$$
\begin{aligned}
(\hbar \nabla)^{\alpha} \psi(\boldsymbol{r}, t) & =-\frac{1}{(2 \pi \hbar)^{3}} \int \mathrm{d}^{3} p \mathrm{e}^{i p r / \hbar}|\boldsymbol{p}|^{\alpha} \varphi(\boldsymbol{p}, t), \\
\varphi(\boldsymbol{p}, t) & =\int \mathrm{d} \boldsymbol{r} \mathrm{e}^{-i p r / \hbar} \psi(\boldsymbol{r}, t),
\end{aligned}
$$

and $D_{\alpha}$ is the generalized coefficient, the physical dimension of which is $\left[D_{\alpha}\right]=\mathrm{erg}^{1-\alpha} \cdot \mathrm{cm}^{\alpha} \cdot \mathrm{s}^{-\alpha}$. Thus fractional Hamiltonian can be written in the form

$$
\hat{H}_{\alpha}=D_{\alpha}(\hbar \nabla)^{\alpha}+V(\boldsymbol{r}, t) .
$$

This type of Hamiltonian has been used to describe the specific heat of noncrystalline solids (glasses) associated with the unusual structure of these materials [39].

\subsection{Galilean noninvariance of fractional Schrödinger equation}

Let us consider transformation of eq. (32) when an transition to another inertial frame of reference frame moving with the velocity $v$ is occurred. Under such transformation the spatial coordinates and time are changed as $r^{\prime}=r-v t, t^{\prime}=t$. Then time and fractional spatial derivative is rewritten as

$$
\begin{aligned}
\partial_{t} f & =\left(\partial_{t^{\prime}}-v \partial_{r^{\prime}}\right) f, \\
\left(\hbar \nabla_{r}\right)^{\alpha} & =\left(\hbar \nabla_{r^{\prime}}\right)^{\alpha} .
\end{aligned}
$$

Let us assume that in the resting reference frame $K$ fractional Schrödinger equation has the form

$$
i \hbar \frac{\partial \Psi(\boldsymbol{r}, t)}{\partial t}=D_{\alpha}(\hbar \nabla)^{\alpha} \Psi(\boldsymbol{r}, t) .
$$

While changing the frame of reference to the moving one $K^{\prime}$, old wave function is written as [40]

$$
\Psi(\boldsymbol{r}, t)=\varphi\left(\boldsymbol{r}^{\prime}, t^{\prime}\right) \mathrm{e}^{i\left(m v \boldsymbol{r}^{\prime} / \hbar+E t^{\prime} / \hbar\right)},
$$

where $\varphi$ denote wave function in new reference frame and $E=m v^{2} / 2$. It is easy to show that fractional derivative of product is

$$
(\hbar \nabla)^{\alpha} f(x) g(x)=\frac{1}{(2 \pi \hbar)^{2}} \int \mathrm{d} q \mathrm{~d} p|p+q|^{\alpha} \mathrm{e}^{i(p+q) x / \hbar} \hat{f}(p) \hat{g}(q) .
$$

Let us substitute eq. (39) in eq. (38) and apply expression for fractional derivative of product (40). With taking into account that Fourier transform of exponential multiplier is proportional to Dirac delta function $\delta(q-m v)$ one obtains fractional Schrödinger equation in new reference frame in form

$i \hbar \partial_{t^{\prime}} \varphi-i \hbar v \nabla_{r^{\prime}} \varphi+\left(m v^{2}-E\right) \varphi=\frac{D_{\alpha}}{2 \pi \hbar} \int \mathrm{d} p \mathrm{e}^{i p r / \hbar} \hat{\varphi}(p)|p+m v|^{\alpha}$

In that case $E=D_{\alpha}|m v|^{\alpha}$. For Galilean invariance of eq. (41) one has to keep only time derivative and spatial fractional derivative of $\operatorname{order} \alpha$. Only value $v=0$ keep form of FSE. It leads to

$$
\begin{aligned}
r^{\prime} & =r, \\
t^{\prime} & =t,
\end{aligned}
$$




$$
\Psi^{\prime}=\Psi
$$

Thus, fractional Schrödinger equation is Galilean noninvariant, and its form is changed at transition into another inertial frame of reference. As a consequence one needs to choose some special reference frame where, for example, nanoporous media is in a rest as well as to introduce additional potential into two-fluid hydrodynamic model.

\section{Conclusion}

It was shown that fractionalized set of hydrodynamical equations with taking into account nonextensivity of He-II inside nanopores leads to coupling between the first and the second sounds that appears even in the absence of viscous friction, which is undoubtedly the effect of fractional dimensionality of nanopore space and nonextensive nature of helium droplets. It was proposed that for the microscopical description of superfluid in nanoporous media with complex fractal structure one can use the fractional Schrödinger equation. But it is necessary to keep in mind that the fractal geometry of nanoporous media leads to the Galilean noninvariance of this equation and as a consequence one needs to choose the special frame of reference where, for example, nanoporous media is in a rest. In the framework of phenomenological two-fluid hydrodynamic model the fractal geometry forces to introduce additional potentials for adequate description of the properties of quantum liquids inside nanoporous media.

This work was supported in part by the Russian Fund for the Fundamental Research (09-02-01253) and the Ministry of Education and Science of the Russian Federation (FTP "Scientific and scientific-pedagogical personnel of the innovative Russia” contract N 02.740.11.0797).

1 Naletov V V, Tagirov M S, Tayurskii D A. Magnetic coupling between liquid $3 \mathrm{He}$ and solid insulators (Review). Low Temp Phys, 2002, 28: $431-448$

2 Minnillin A R, Tayurskii D A. Magnetic susceptibility of noninteracting fermions in a confined geometry. JETP Lett, 2000, 72: 616-620

3 Tagirov M S, Yudin A N, Mamin G V, et al. Nuclear spin-kinetics of He-3 in carbonizates with various porosity. J Low Temp Phys, 2007, 148: $815-819$

4 Klochkov A V, Kuz'min V V, Safiullin K R, et al. Nuclear magnetic relaxation of ${ }^{3} \mathrm{He}$ in contact with an aerogel above the Fermi temperature. JETP Lett, 2008, 88: 823-827

5 Wong G, Crowell P, Cho H, et al. Superfluid critical behavior in $4 \mathrm{He}$ filled porous media. Phys Rev Lett, 1990, 65: 2410-2413

6 Matsumoto K, Tsuboya H, Yoshino K, et al. Possible sound mode conversion in superfluid ${ }^{4} \mathrm{He}-97 \%$ open aerogel system. J Low Temp Phys, 2007, 148: 615-620

7 Vasquez C, Paredes R. Effects of aerogel-like disorder on the critical behavior of $\mathrm{O}(\mathrm{m})$-vector models. Recent simulations and experimental evidences. Cond Matt Phys, 2006, 9: 305-317

8 Azuah R T, Glyde H R, Scherm R, et al. Bose-Einstein Condensation in liquid ${ }^{4} \mathrm{He}$ in Vycor. J Low Temp Phys, 2003, 130: 557-567

9 Tayurskii D, Lysogorskii Y, Zvezdov D. Two-fluid hydrodynamic model for superfluids in fractal dimensions. J Phys-Conf Ser, 2009, 150: 032110
10 Tayurskii D A, Lysogorskii Y V. Nonextensive entropy of quantum liquid in fractal dimension space. J Low Temp Phys, 2009, 158: 237-243

11 Bossy J, Pearce J V, Schober H, et al. Phonon-Roton modes and localized Bose-Einstein condensation in liquid helium under pressure in nanoporous media. Phys Rev Lett, 2008, 101: 025301

12 Wada N, Matsushita T, Hieda M, et al. Fluid states of helium adsorbed in nanopores. J Low Temp Phys, 2009, 157: 324-351

13 Kanamori K, Aizawa M, Nananishi K, et al. New transparent methylsilsesquioxane aerogels and xerogels with improved mechanical properties. Adv Mater, 2007, 19: 1589-1593

14 Khalatnikov I M. An Introduction to the Theory of Superfluidity. Redwood City: Addison-Wesley, 1988

15 Tilley D, Tilley J. Superfluidity and Superconductivity. 3rd ed. London: IOP Publishing Ltd, 1990

16 Coste C. Nonlinear Schrodinger equation and superfluid hydrodynamics. Eur Phys J B, 1998, 253: 245-253

17 Hills R N, Roberts P H. Healing and relaxation in flows of helium II. Part II. First, second, and fourth sound. J Low Temp Phys, 1978, 30: 709-727

18 Ginzburg V L, Pitaevskii L P. On the theory of superfluidity. Sov Phys JETP, 1958, 7: 858-861

19 Khalatnikov I M. Absorption and dispersion of sound in a superfluid liquid near the lambda-point. Sov Phys JETP, 1970, 30: 268-272

20 Hills R N, Roberts P H. Healing and relaxation in flows of helium II: Generalization of landau's equations. Int J Eng Sci, 1977, 15: 305-316

21 Guerst J. General theory unifying and extending the LandauKhalatnikov, Ginzburg-Pitaevskii, and Hills-Roberts theories of superfluid ${ }^{4}$ He. Phys Rev B, 1980, 22: 3207

22 Ristig M L, Gernoth K A. Statistical properties of strongly correlated quantum liquids. Found Phys, 2009, 40: 1253-1262

23 Mongiovi M S. Extended irreversible thermodynamis of liquid helium II. Phys Rev B, 1993, 48: 6276-6283

24 Mongiovi M S, Peruzza R A. Velocity of the fourth sound in liquid helium II via extended thermodynamics. Math Phys, 2003, 54: 566-583

25 Porto J, Parpia J. Correlated disorder in a p-wave superfluid. Phys Rev B, 1999, 59: 14583-14592

26 Courtens E, Vacher R. Structure and dynamics of fractal aerogels. Z Phys B Cond Matt, 1987, 68: 355-361

27 Hasmy A, Anglaret E, Foret M, et al. Small-angle neutron-scattering investigation of long-range correlations in silica aerogels: Simulations and experiments. Phys Rev B, 1994, 50: 6006-6016

28 McKenna M J, Slawecki T M, Maynard J D. Second and fourth sound modes for superfluid helium in aerogel. Physica B, 1990, 165-166: 581582

29 McKenna M J, Slawecki T M, Maynard J D. Observation of a secondsound-like mode in superfluid-filled aerogel. Phys Rev Lett, 1991, 66: 1878

30 Biot M A. Theory of propagation of elastic waves in a fluid-saturated porous solid. J Acoust Soc Am, 1956, 28: 168-178

31 Feder E. Fractals. Berlin: Springer, 2007

32 Gell-Mann M, Tsallis C. Non-extensive Entropy - Interdisciplinary Applications. New York: Oxford University Press, 2004

33 Parvan A, Biro T. Thermodynamical limit in non-extensive and Renyi statistics. arXiv: cond-mat/0607190, 2006

34 Badescu V. Physical temperature and pressure in fully nonextensive statistical thermodynamics. Adv Comp Syst, 2008, 11: 43

35 Wang Q A, Nivanen L, Mehaute A L, et al. On the generalized entropy pseudoadditivity for complex systems. J Phys A-Math Gener, 2002, 35: 7003-7007

36 Abe S. General pseudoadditivity of composable entropy prescribed by the existence of equilibrium. Phys Rev E, 2001, 63: 061105

37 Abe S, Martinez S, Pennini F, et al. Nonextensive thermodynamic relations. Phys Lett A, 2001, 281: 126-130

38 Laskin N. Fractional Schrodinger equation. Phys Rev E, 2002, 66: 56108

39 Lenzi E K, Oliveira B F, Astrath N G, et al. Fractional approach, quantum statistics, and non-crystalline solids at very low temperatures. Eur Phys J B, 2008, 62: 155-158

40 Greenberger D. Inadequacy of the usual Galilean transformation in quantum mechanics. Phys Rev Lett, 2001, 87: 1-4

Open Access This article is distributed under the terms of the Creative Commons Attribution License which permits any use, distribution, and reproduction in any medium, provided the original author(s) and source are credited. 